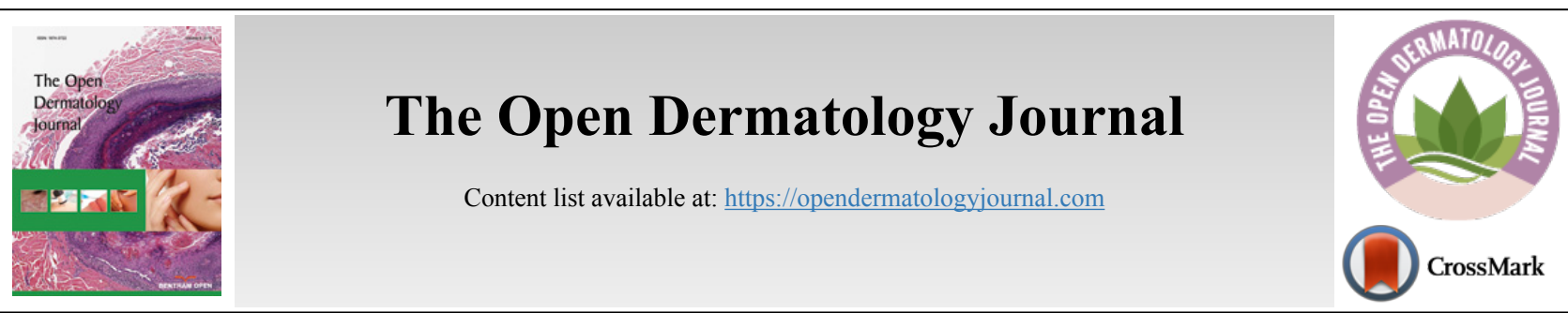

RESEARCH ARTICLE

\title{
Development and Evaluation of Herbal Hair Serum: A traditional way to Improve Hair Quality
}

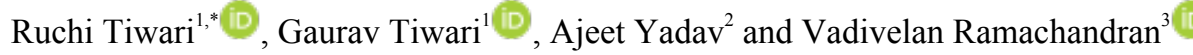 \\ ${ }^{1}$ Department of Pharmacy, Pranveer Singh Institute of Technology, Kanpur, India \\ ${ }^{2}$ Department of Pharmaceutical Sciences, J.S. University, Shikohabad, Firozabad-283135, India \\ ${ }^{3}$ Department of Pharmacology, JSS College of Pharmacy, JSS Academy of Higher Education \& Research, Ooty, Nilgiris, Tamil Nadu, India
}

\begin{abstract}
:
Background:

In the mammalian system, the hair follicle is known to be the most significant organ that determines appearance, gender distinction, provides intense temperature protection, and plays a role in self-defense. The younger generations have begun to suffer from extreme hair loss problems due to many lifestyle-related changes such as fatigue, anxiety, intake of junk foods, use of different hairstyling/coloring methods, etc. The loss of hair is not temporary in most cases, but it results in alopecia. Many people suffering from hair loss are in search of multiple treatments due to extreme anxiety and tension, from mythology to traditional and therapeutic healing to the use of minoxidil and finasteride. To improve hair growth and to prevent hair loss, hair root activation is required.
\end{abstract}

\section{Objective:}

The present study was intended to use different herbs to formulate herbal hair serum for general purposes (hair application).

Methods:

Crude herbs are obtained from nearby regions. In order to obtain the extract, the required part of herbs, such as Citrus sinensis peel, Zingiber officinale roots, and Linum usitatissimum seeds, Nigella sativa, and Trigonella foenum-graecum were specifically weighed and dispensed in water, boiled, allowed to cool, and then filtered. To the filtrate, castor oil and vitamin E were added. The formulated herbal hair serum was tested, and different criteria were determined and recorded in this text, such as physical appearance, viscosity, $\mathrm{pH}$, homogeneity, eye sensitivity (Draize eye test), hair growth activity, hair weight, stability test, etc.

\section{Results:}

Prepared herbal hair serum was found to be pale brown in color with pseudoplastic behavior. The texture of the formulation was smooth and lubricative, and the $\mathrm{pH}$ was within the acceptable limit. Herbal hair serum revealed good hair growth as well as hair weight after application with minor sensitivity for the first time. The formulation was found to be stable for seven days.

\section{Conclusion:}

Herbal cosmetics are still commonly used by average citizens because of fewer side effects and greater protection and safety profile. Current research has revealed that herbal formulations are effective in enhancing hair consistency.

Keywords: Citrus Sinensis, flaxseed, Nigella sativa, Zingiber officinale, castor oil, Trigonella foenum-graceum.

\section{Article History} Received: March 1, 2021 Revised: April 2, 2021

\section{INTRODUCTION}

Hair growth is based on a dynamic and precisely regulated mechanism that is not fully understood. It is a cyclical mechanism involving hair shaft synthesis, elongation, and ultimately

\footnotetext{
"Address correspondence to this author at the Department of Pharmacy, Pranveer
} Singh Institute of Technology, Kanpur, India; E-mail: tiwaridrruci@gmail.com shedding. Human hair usually comprises follicles of anagen, catagen, and telogen phases. The hair follicle in the anagen phase actively accumulates cytochrome and easily develops the hair shaft. When the hair follicle matures into the anagen hair follicle, the telogen hair follicle is not able to develop neonatal hair shafts [1]. Alopecia Areata (AA) is a recurrent immunemediated skin disorder with a non-scarring loss of hair. In the 
general population, it has an incidence range of 1.7 percent and 2.1 percent, with higher prevalence in young patients (21 40 years) and no significant difference in incidence between males and females reported. This condition may have a detrimental effect on the quality of life of patients in a manner close to that reported for other skin diseases such as dermatitis and psoriasis. AA's pathogenesis can be explained by the inflammatory cascades involving a breakdown of the hair follicle's immune privilege, associated with $\mathrm{T}$ lymphocyte invasion, and an autoimmune process that creates autoantigens from proteins associated with melanogenesis. In addition, different variables, including family backgrounds, genetic bases, environmental factors, illnesses, medications, injuries, potential emotional stress, and oxidative stress, can be implicated in AA pathogenesis [2]. Defective antioxidant defense or exaggerated expression of reactive oxygen species (ROS) has been reported to predispose to oxidative stress, which is believed to play a significant role in many inflammatory skin disorders such as AA. In this respect, several studies have documented that $\mathrm{AA}$ has a detrimental effect on the blood and skin tissue's oxidant-antioxidant balance. AA's latest clinical treatments are centered primarily on immune regulation using numerous forms of medications, including topical and systemic steroids, phototherapy, and immune suppressants such as cyclosporine and methotrexate; meanwhile, certain vitamins and micronutrients are expected to play a role in the treatment of AA and can alleviate oxidative stress [3].

Citrus sinensis (orange peel) has shown remarkably positive results on hair. Daily use can make the hair smoother and shinier with these peels. They are filled with antioxidants and help to treat the harm caused by pollutants as well. Daily use of orange peels, filled with a lot of vitamin C, makes the hair bouncy and shiny and reduces dryness and dullness of hair. In addition, orange peels, a huge source of vitamin B12 and vitamin $\mathrm{E}$, assist in hair development. They also delay the pace at which the hair begins to turn gray $[4,5]$.

The richest plant source of the $\omega-3$ fatty acid is flaxseed, i.e., " $\alpha$ - linolenic acid" (ALA). Flaxseed oil is low (9\%) in saturated fatty acids, high (18\%) in monosaturated fatty acids, and rich in polyunsaturated fatty acids (73\%) [6]. The possible health effects of flaxseed oil, fibers, and flax lignans include minimizing coronary disease, atherosclerosis, asthma, cancer, obesity, osteoporosis, autoimmune and neurological disorders. In addition, flaxseed is full of fatty acids and antioxidants that tend to remove pollutants and dead cells from the scalp. Flaxseed gel can be added as a moisturizer to the scalp and hair, which can help promote growth and boost the quality of the current hair. Flaxseed gel is super hydrating, has conditioning benefits, and, without the crunch, makes the hair very fluffy $[7,8]$.

Nigella sativa (NS) is an annual flowering plant native to the Mediterranean countries, Pakistan, and India, commonly known as black cumin. The key pharmacologically active components of NS are thymoquinone (TQ), dithymoquinone (DTQ), thymohydroquinone (THQ), and thymol (THY) [9]. Inhibition of NF-kB activation has been reported to inhibit inflammatory cell infiltration in the brain by $\mathrm{TQ}$, which constitutes the predominant portion $(30 \%-48 \%)$ of NS. By inhibiting cyclooxygenase 2 (COX-2) expression and prostaglandin D2 (PGD2) synthesis, TQ demonstrates antiinflammatory behavior, which will explain why NS has been considered a natural treatment for many illnesses and inflammatory disorders [10]. In addition, recent studies have shown that PGD2 has a significant inhibitory function in the hair follicle. In particular, its binding to the GPR44 receptor expressed in the hair follicle seems to cause follicle miniaturization. This contributes to the growth and preservation of vellus-like hair and is likely to inhibit stem cells from maturing into progenitor cells and vellus-like hair from maturing into natural, dense, and pigmented, terminal hair. Consequently, NS inhibition of NF-kB and PDG2 activation and synthesis can contribute to the regulation of the hair cycle. Nigella sativa, a flower native to Eastern Europe, Southwest Asia, and the Middle East, contains black seeds. Black caraway, black cumin, black onion seeds, and kalonji are widely recognized. These seeds have been used in treatments for allergy, asthma, diabetes, headache, weight loss, arthritis, and intestinal worms for thousands of years. Nigella sativa seed is an ideal ingredient for medicine and cosmetics, according to a 2016 study. The research identifies the properties of black seed oil as antibacterial, antifungal, anti-inflammatory, and antioxidant. Advocates of black seed oil for hair claim that by treating concerns such as dandruff while maintaining the scalp moisturized, these properties can help in protecting the scalp. Since it is anti-inflammatory, when diluted with a carrier oil to soothe scalp conditions that cause inflammation, flakiness, and discomfort, the black seed oil is perfect [11]. It also destroys fungal infections and viruses, so it can help people with lice. Black seed oil is an adjuvant, like lavender, lemon, and rosemary essential oils, ensuring it combines well with other ingredients to exhibit medicinal effects. Black seed oil is antirheumatic, and it is very helpful in alleviating joint injuries that arise with age and athleticism. Furthermore, black seed oil can help to regrow hair in thinning regions on the scalp. Nigella sativa oil has commonly been used for thousands of years for the treatment of hair loss [12].

Fenugreek seed extract contains a dietary supplement that also contains micronutrients such as B-vitamins, antioxidants, and trace elements that are also present in the hair. Fenugreek (Trigonella foenum-graceum L.) is a leguminous herb. Fenugreek is native to Southern Europe, Western Asia, and the Mediterranean region. Fenugreek seeds contain a wide range of active ingredients such as saponins, especially diosgenin, yamogenin, and gitogenin derivatives, trigonelline alkaloids, flavonoids, galactomannan vitamins, and fiber, etc [13]. The seeds have a good fragrance and a sour flavor. As both a culinary and medicinal plant of the ancient world, it has a long tradition. Because of their therapeutic ability, including their usage as hypoglycemic, antiulcerogenic, hypo-cholesterolemic, and antihypertensive agents, the beneficial properties of fenugreek seeds have drawn significant interest in the recent past [14]. There are known beneficial effects of fenugreek on hair growth, but the mechanism is not characterized. It is addressed that fenugreek interacts in a physiological manner with the synthesis of DHT (dihydrotestosterone) by enhancing the blood supply of hair follicles and steroid saponins. The 
impact of DHT on genetically predisposed hair follicles is suspected to be one cause for male and female variant hair loss. Binding DHT to the hair follicle results in the hair's progressive miniaturization and subsequent hair loss $[15,16]$.

Ginger, one of the most commonly used spices worldwide, is the root of Zingiber officinale (L.) Rosc. It contains several active ingredients, including gingerol, zingerone, shogaol, and beta bisabolene. It has been used in various diseases such as rheumatoid arthritis, neurodegenerative diseases, inflammation, and asthma as folk medicine. In addition to high antioxidant activity and reduced expression of certain pro-inflammatory biomarkers, many of its active constituents have anti-diabetes, anti-cancer, and anti-inflammatory functions. In addition, ginger is helpful in the treatment of dandruff and irritated, itchy scalp. It has natural anti-inflammatory and antiseptic properties that tend to keep the skin clean and healthy. Overall, ginger stimulates the growth of hair, prevents hair from thinning, and renders hair to be glossy and smooth $[17,18]$.

Castor oil, also known as Ricinus communis, is a vegetable oil that is rich in nutrients and comes from castor beans. While it originates from tropical East Africa, it is commonly used in India and the West Indies for cosmetic and medicinal purposes. Castor oil, which is known as black castor oil, is typically transparent or pale yellow unless it is derived from roasting or boiling. Castor oil is a long-chained fatty acid at the molecular level. "Fatty acids give hair follicles essential proteins and nutrients and prevent inflammation of hair follicles," as according to Bridgette Hill. Castor oil has a few other noteworthy effects in addition to being anti-inflammatory. "Increased gloss or shine of the hair, increased hair shaft flexibility by coating the hair shaft, and antimicrobial properties are some of the benefits of using castor oil." A total of $90 \%$ of castor oil is made up of ricinoleic acid fatty acid. It is believed to activate the Prostaglandin E2 receptor, which can contribute to the dilation of the blood vessels. Increased blood vessel dilation increases the supply of oxygen-rich and nutrient-rich blood to the follicle's powerhouse, dermal papilla, or hair root [19].

The present study intended to use extracts of different herbs to formulate herbal hair serum for the improvement of hair quality.

\section{MATERIALS AND METHODS}

\subsection{Collection of Plant Part}

For the preparation of herbal hair oil, various plant materials were collected viz., Citrus sinensis, Linum usitatissimum, Nigella sativa, Zingiber officinale, and Trigonella foenum-graecum from the Medicinal Plant Garden of Pranveer Singh Institute of Technology, Kanpur, Uttar Pradesh, India, and were properly authenticated in the Department of Pharmacognosy.

\subsection{Formulation of Herbal Hair Serum}

Table 1 presents the different ingredients used in the formulation of herbal hair serum. All fresh herbs, such as Citrus sinensis peel, Zingiber officinale roots, Linum usitatissimum seeds, and Nigella sativa, Trigonella foenumgraecum were specifically weighed and dispensed in $500 \mathrm{ml}$ of water. The contents referred to above were boiled for $15 \mathrm{~min}$. They were permitted to cool after 15 minutes of boiling and then filtered. Castor oil and vitamin E were added to the filtrate. Afterward, the Prepared serum was kept in a spray bottle.

\subsection{Evaluation of The Hair Serum}

\subsubsection{Physical Appearance}

The physical appearance, color, and feel of the prepared herbal hair serum are visually tested. Table 2 reflects the outcomes.

\subsection{Homogeneity Test}

A clean and dry object glass was smeared with the hair serum, and a cover glass was sealed. The appearance under the light of some coarse particle/homogeneity was investigated. Herbal hair serum was tested by visual examination for homogeneity and tested for some lumps, flocculates, or aggregates [20].

\section{5. pH Test}

The $\mathrm{pH}$ meter was calibrated using $\mathrm{pH} 4$ and $\mathrm{pH} 7$ buffer solutions. Then, the electrode was soaked in the hair serum and left until the $\mathrm{pH}$ normalized after a few minutes [21].

\subsection{Viscosity}

The viscosity measurement was performed with spindle number 6 on a Brookfield viscometer (RVDV-II+PRO). In the beaker, $50 \mathrm{ml}$ of hair serum was placed, and the viscosity was measured at various rpm, i.e., 10, 20, 50, 100 [20].

\subsection{Spreadability}

Spreadability was measured by a parallel plate process typically used to assess and measure the spreadability of semisolid preparations. One gram hair serum was pressed between two horizontal plates of dimension $20 \times 20 \mathrm{~cm}$, the upper of which weighed $125 \mathrm{~g}$. The spread diameter was measured after 1 min. Spreadability was calculated using the following formula:

$$
\mathrm{S}=\mathrm{M} \times \mathrm{L} / \mathrm{T}
$$

Where, $\mathrm{S}=$ Spreadability, $\mathrm{M}=$ Weight in the pan (tied to the upper slide), $\mathrm{L}=$ Length moved by the glass slide, and $\mathrm{T}=$ Time (in sec) taken to separate the slides completely [21].

Table 1. Ingredients used in the preparation of herbal hair serum.

\begin{tabular}{|c|c|c|}
\hline Ingredients & Plant part & Quantity (\%) \\
\hline Citrus sinensis & Peel of fruit & 70 \\
Zingiber officinale & Root & 5 \\
Linum usitatissimum, & Seed & 5 \\
Nigella sativa, & Seed & 8 \\
Trigonella foenum-graecum & Seed & 10 \\
Castor oil & - & 0.5 \\
Vitamin E & - & 1.5 \\
\hline
\end{tabular}


Table 2. Evaluation parameters of herbal hair serum.

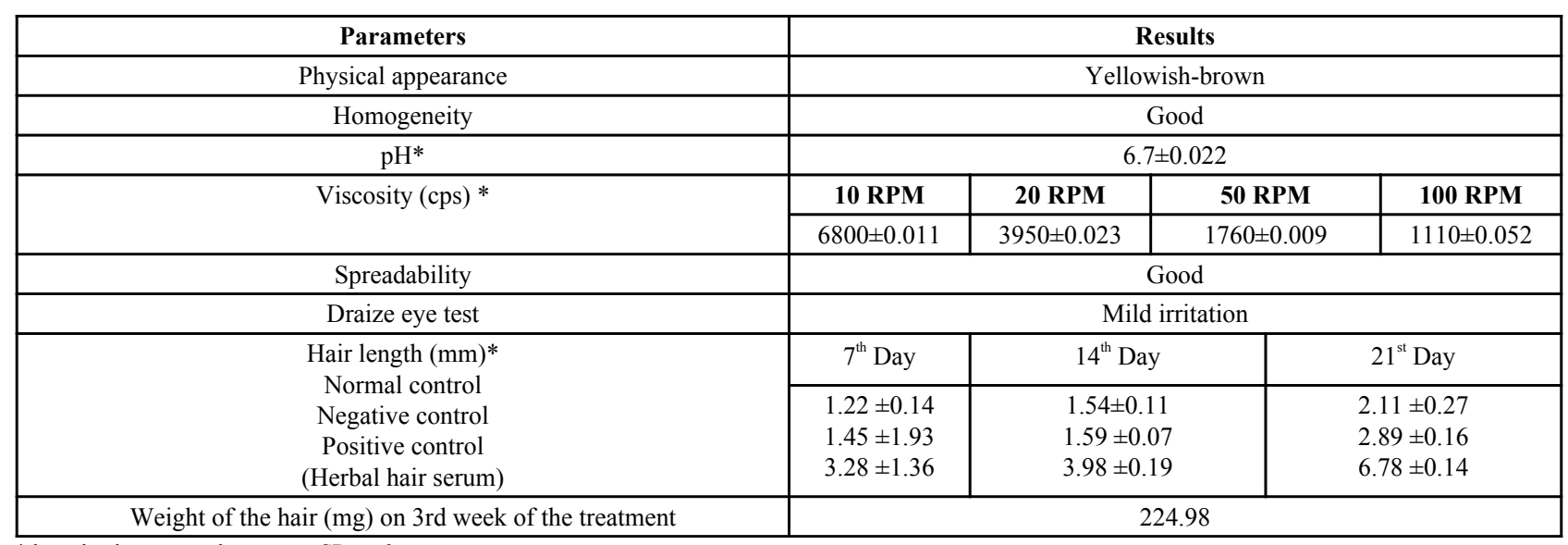

*the value is expressed as mean $\pm \mathrm{SD}, \mathrm{n}=3$.

Table 3. Stability study on herbal hair serum.

\begin{tabular}{|c|c|c|c|}
\hline \multicolumn{2}{|c|}{ pH* } & \multicolumn{2}{c|}{ Viscosity at 100 rpm (centipoise) * } \\
\hline Initial & Final & Initial & Final \\
\hline $6.7 \pm 0.022$ & $6.6 \pm 0.012$ & $1110 \pm 0.002$ & $1124 \pm 0.117$ \\
\hline
\end{tabular}
*the value is expressed as mean $\pm S D, n=3$.

\subsection{Test the Sensitivity of the Eye (Draize Eye Test)}

Eye sensitivity was tested on three rabbits for getting the average result of sensitivity. One drop of hair serum was injected into the left eyes of the rabbits in physiological $\mathrm{NaCl}$ (as the control is the right eye) and then observed for 30 minutes, 60 minutes, 120 minutes, 240 minutes, 1 day, 2 days, 3 days, and 4 days. The scores of the cornea, iris, and conjunctiva were determined [21].

\subsection{Hair Growth Activity Test}

This test was conducted on 3 areas of a single rabbit on either foot, right and left of the shaved backs of the rabbits, each $4 \times 4 \mathrm{~cm}$ in size. Then, after 3-5 minutes, depilatory cream (Veet ${ }^{\circledR}$ cream) was applied, and the area was rinsed with water until the area was clean with hair. As an antiseptic, 70 percent ethanol was then added. Before any action evaluation, the rabbit was left behind for 24 hours. As no intervention was performed, treatment 1 was the standard control, and treatment 2 was the positive control where the hair serum with the test material was added. Again, as no intervention was undertaken, treatment 1 was the standard control, and treatment 2 was the positive control where hair serum with the test material was added. For 3 weeks, $0.1 \mathrm{ml}$ of each drug was then administered twice a day to the rabbit. The first day of use of the hair serum was called day $0[5,6]$.

\subsection{Qualitative Assessment of Hair Growth}

Qualitative measurement of hair growth analysis was carried out by visual inspection of 2 criteria, initial hair growth time (minimum time needed for hair growth on the shaved area, measured from the darkening of the skin color showing initial hair growth) and completion time for hair growth (minimum time required for the entire shaved area to be covered with new hair) [22] on three groups (Group I: Normal control; Group II: Negative control; Group III: Positive control (Herbal hair serum)). Each group consists of three rabbits.

\subsection{Observations of the Growth of Hair}

It was done by taking 10 random hair strands on each box on days 7, 14, and 21. The hair had been drawn out, straightened, and stuck with the tape, and measured using Mitutoyo Digimatic wireless calipers. The average length collected was analyzed to see if the difference between the research region and the control was statistically significant $[4$, 21].

\subsection{Hair Weight Measurements}

To evaluate the weight of each box, the hair was removed and weighed on day 21 and then statistically measured $[3,20]$.

\subsection{Microbial Contamination}

Microbial contamination of hair serum was determined via spreading a thin loopful of the serum on a nutrient and Sabouraud agars and incubating for 48 hours at $37^{\circ} \mathrm{C}$. In order to examine the degree of contamination, one gram of material was used to be dispersed in $4 \mathrm{ml}$ of sterile Ringer solution, containing $0.25 \%$ Tween 80 . In the same dispersion vehicle, sufficient dilutions were made, and $0.5 \mathrm{ml}$ was mounted on the appropriate solid medium using the viable surface procedure. Following required incubation, emergent colonies were counted [23, 24].

\subsection{Stability}

The herbal hair serum was kept for three months at two separate temperatures of $4 \pm 2^{\circ} \mathrm{C}$ and $30 \pm 2{ }^{\circ} \mathrm{C}$, with $65 \% \mathrm{RH}$. Compared with the original $\mathrm{pH}$ and viscosity, the $\mathrm{pH}$ and viscosity of the herbal hair serum were determined after three months [21].

\section{RESULTS AND DISCUSSION}

\subsection{Physical Appearance}

It was observed that the color of all the herbal hair serum was pale brown with a translucent look, which on the application was found to be smooth. 


\subsection{Homogeneity}

By visual examination of the appearance and presence of any lumps, flocculates, or aggregates, the produced herbal hair serum was checked for homogeneity. The homogeneity of prepared serum has been shown to be fine.

\subsection{Rheological Study}

The rheological examination of prepared herbal hair serum was conducted, and it was observed that viscosity was reduced as the shear rate increased (Fig. 1). The pseudoplastic flow was also described, which is a beneficial attribute for topical herbal hair serum since it guarantees optimum area coverage upon application. The mechanistic explanation for the flow behaviour found is as follows: the long-chain molecules of the polymers are spontaneously arranged in dispersion under standard storage conditions. These molecules tend to organize their long axes in the direction of the force exerted when applying shear stress. This stress-induced orientation reduces the internal resistance of the material. In addition, the solvent molecules earlier associated with the polymer molecules will also be released. Thus, the effective concentration and size of the molecules are low. The material allows a progressive increase in the shearing stress [19].

\section{4. pH Determination}

The $\mathrm{pH}$ of the whole herbal hair serum was 7.3 , which was sufficient for the hair, suggesting that the herbal hair serum was suitable for the hair [19].

\subsection{Draize Eye Test}

To evaluate the protection of the herbal hair serum, a sensitivity assessment test was performed. To determine the ocular sensitivity index, the test was conducted on 3 rabbits. Tests on the left eye were conducted with controls on the right eye. In physiological saline, 3 drops of sterile 2.5 percent herbal extract were added to the left eye of the rabbit, and measurements were taken at 30 minutes, 60 minutes, 120 minutes, 240 minutes, 1 day, 2 days, 3 days, 4 days. There was no image of opacity in the left eye, normal conjunctiva, no swollen eyelid, and the iris image appeared normal. It was seen that the eyes created tears. The index of ocular annoyance was 2 on the first day and 0 on the second, third, and fourth days (maximum ocular irritation index of 110). Thus, it can be inferred that the herbal serum possesses a moderate inflammatory effect [21].

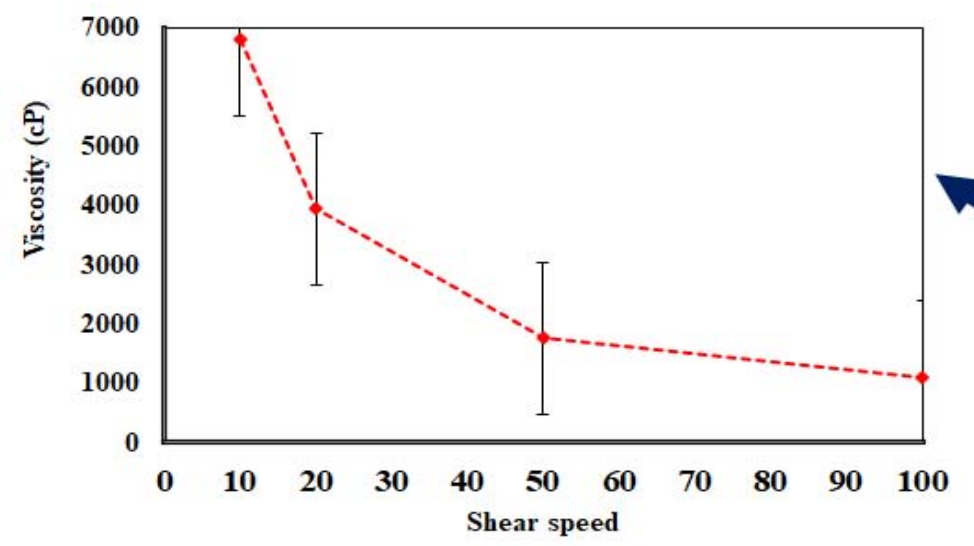

\section{B. Viscosity of prepared herbal hair serum}
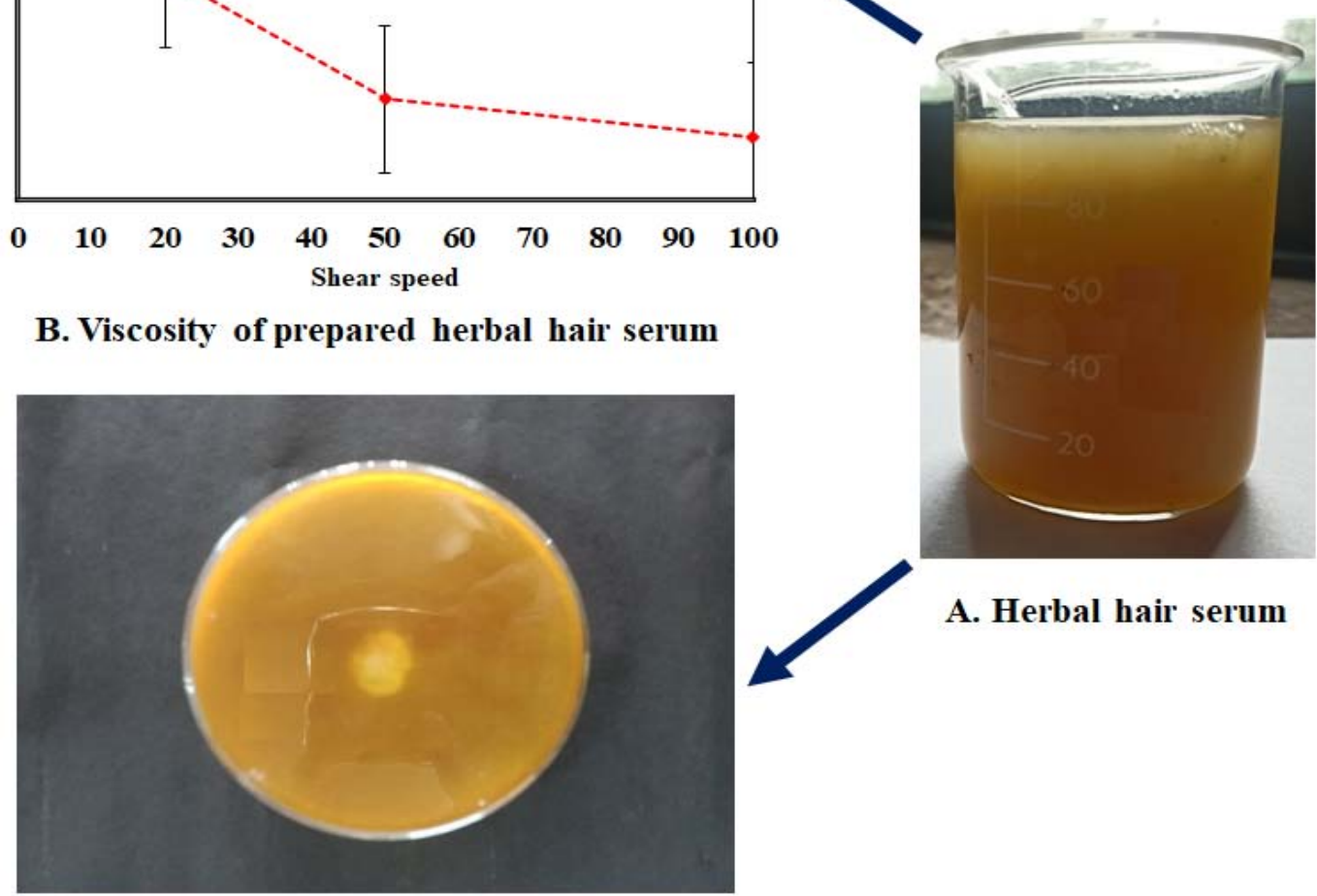

A. Herbal hair serum

C. Microbial contamination study

Fig. (1). 7 KHUKRRI IFDOH DP IQDURQRRILSUSDHGIKHEDOKDUUUHXP . 


\subsection{Hair growth activity test}

Table 2 reveals that normal control showed a very close outcome with negative control for the first week, while positive control showed little difference. However, in areas treated with positive regulation, there is a substantial hair length difference between both normal control and negative control hair. However, a major difference was reported by comparing the growth behavior displayed by normal control, negative control, and positive control. The discrepancy between the groups was found to be important $(\mathrm{p}<0.05)$. Thus, it was found that, although using a single formula, the hair serum used in this study displayed a more important hair growth activity of 6.98 $\mathrm{mm}$. In Table 2, it can be seen that in the third week, herbal hair serum induced a greater weight of the hair [21, 22].

\subsection{Microbial Contamination}

At the end of seven days, the microbial contamination of the herbal hair serum after 24 hours was observed to be 1.89 CFU for fungi [23].

\subsection{Stability Studies}

Table 3 reveals that the herbal hair serum was stable during the research time, as these serums showed no physical instability, and there was no noticeable difference in the $\mathrm{pH}$ before and after the study [23, 24].

\section{CONCLUSION}

Results have shown that herbal hair serum provides various essential nutrients needed to preserve the proper function of the sebaceous glands and support the growth of natural hair. In the personal hygiene and health care system, the use of herbal cosmetics has changed by several folds. Therefore, the herbal cosmeceutical individual care or personal health care industry, which is actually concentrating and paying extra care on the production of herbal-based cosmetics, has a considerable clamor. As nowadays, in the coming years, it is a fastdeveloping market with a mammoth scope. In cosmetic formulations, the use of bioactive ingredients has a valuable impact on body characteristics and offers nutrients that are important for preserving good and beautiful hair. It can be inferred that prepared herbal hair serum has a beneficial effect on the mechanism of hair growth and increased consistency. Medicinal plants have been used for the treatment of hair diseases since antiquity because of fewer side effects and hypersensitivity reactions. It is time to dump the chemicalladen hair care products in favour of natural alternatives. The traditional system of medicine in India acclaims a number of herbal drugs for hair growth promotion. The best part is that herbal extracts will provide microprotein supplements to hair and provide enough nourishment, resulting in safe and sound hair. Herbal cosmetics have become increasingly common in the personal care industry, and there is a high demand for them in everyday life due to their lack of parabens and sulphates. The global herbal industry is projected to be worth more than US\$10 billion dollars, and it is increasing at a rate of three to four percent per year due to increased demand. In terms of production and demand of herbal products, Europe is the largest region, followed by Asia.

\section{ETHICS APPROVAL AND CONSENT TO PARTICIPATE}

Not applicable.

\section{HUMAN AND ANIMAL RIGHTS}

No animals/humans were used in the studies that are basis of this research.

\section{CONSENT FOR PUBLICATION}

Not applicable.

\section{AVAILABILITY OF DATA AND MATERIALS:}

The data that support the findings of this study are available from the corresponding author [R.T] upon a reasonable request.

\section{FUNDING}

None.

\section{CONFLICT OF INTEREST}

The authors declare no conflict of interest, financial or otherwise.

\section{ACKNOWLEDGEMENTS}

Declared none.

\section{REFERENCES}

[1] Aruna V, Amruthavalli GV, Gayathri R. Hair root activation by anagen grow- a herbal hair growth serum. Dermatol \& Cosmet 2019; 1(3): $56-9$.

[2] Begum R, Begum A. Preparation and evaluation of herbal hair oil. Int J of Res and Anal Reviews 2019; 6(1): 266-9.

[3] Alfredo R, Lara P, Alessandra I, et al. Evaluation of a therapeutic alternative for telogen effluvium: A pilot study. J Cosmetics Dermal Sci App 2013; 3: 9-16.

[4] Erukainure OL, Ebuehi OA, Iqbal Chaudhary M, et al. Orange peel extracts: Chemical characterization, antioxidant, antioxidative burst and phytotoxic activities. J Diet Suppl 2016; 13(5): 585-94. [http://dx.doi.org/10.3109/19390211.2016.1150932] [PMID: 26930349]

[5] Zhang Q. Development of citrus peel ethanolic extract shampoo. IOP Conf Ser: Mater Sci Eng. 1-6. [http://dx.doi.org/10.1088/1757-899X/479/1/012014]

[6] Goyal A, Sharma V, Upadhyay N, Gill S, Sihag M. Flax and flaxseed oil: An ancient medicine \& modern functional food. J Food Sci Technol 2014; 51(9): 1633-53.

[http://dx.doi.org/10.1007/s13197-013-1247-9] [PMID: 25190822]

[7] Halligudi N. Pharmacological properties of Flax seed: A Review. Hygeia J D Med 2012; 4(2): 70-7.

[8] Manjula D, Josephine JLJ, Kumari P, Banu S. Formulation and evaluation of flaxseed hair gel: A natural hair tamer. Int J Res Pharm Chem 2018; 8(3): 487-91.

[9] Saleem U, Sabir S, Ahmad B. Protective role of Nigella sativa in chemotherapy induced alopecia. Bangladesh J Pharmacol 2017; 12: 455-62.

[http://dx.doi.org/10.3329/bjp.v12i4.33552]

[10] Yousefi M, Barikbin B, Kamalinejad M, et al. Comparison of therapeutic effect of topical Nigella with Betamethasone and Eucerin in hand eczema. J Eur Acad Dermatol Venereol 2013; 27(12): 1498-504.

[http://dx.doi.org/10.1111/jdv.12033] [PMID: 23198836]

[11] Ali BH, Blunden G. Pharmacological and toxicological properties of Nigella sativa. Phytother Res 2003; 17(4): 299-305. [http://dx.doi.org/10.1002/ptr.1309] [PMID: 12722128]

[12] Majdalawieh AF, Fayyad MW, Nasrallah GK. Anti-cancer properties and mechanisms of action of thymoquinone, the major active 
ingredient of Nigella sativa. Crit Rev Food Sci Nutr 2017; 57(18): 3911-28.

[http://dx.doi.org/10.1080/10408398.2016.1277971] [PMID: 28140613]

[13] Wijaya WH, Mun'im A, Djajadisastra J. Effectiveness test of fenugreek seed (trigonella foenum-graecum 1.) Extract hair tonic in hair growth activity. Int J Curr Res 2013; 5(11): 3453-60.

[14] Suryawanshi NC, Vijayendra Swamy SM, Nagoba Shivappa N, Wanje VV. Formulation and evaluation of herbal hair gel containing fenugreek seed extract for nourishment and hair growth. Int J Sci Res Sci Technol 2019; 6(4): 92-103.

[15] Schulz C, Bielfeldt S, Reimann J. Fenugreek+micronutrients: Efficacy of a food supplement against hair loss. Cosmetic Medicine 2006.

[16] Didarshetaban MB, Pour S, Reza H. Fenugreek (Trigonellafoenumgraecum L.) as a valuable medicinal plant. Int J Adv Biol Biomed Res 2013; 1: 922-31

[17] Abbas AN. Ginger (Zingiber officinale (L.) Rosc) improves oxidative stress and trace elements status in patients with alopecia areata. Niger J Clin Pract 2020; 23(11): 1555-60.

[http://dx.doi.org/10.4103/njcp.njcp_59_19] [PMID: 33221781]

[18] Mahboubi M. Zingiber officinale Rosc. Essential oil, a review on its composition and bioactivity. Clin Phytosci 2019; 5(6)

[19] Shah RR, Mohite SA, Patel NR. Preparation and evaluation of polyherbal hair oil- an effective cosmetic. Asian J Pharm Res 2018; 8(1): 36-8.

[http://dx.doi.org/10.5958/2231-5691.2018.00007.2]

[20] Gautam S, Dwivedi S, Dubey K, Joshi H. Formulation and evaluation of herbal hair oil. Int J Chem Sci 2012; 10(1): 349-53.

[21] Beroual K, Maameri Z, Halmi S, Benleksira B, Agabou A, Hamdi PY. Effects of Linum usitatissimum L. ingestion and oil topical application on hair growth in rabbit. Int J Med Arom Plants 2013; 3(4): 459-63.

[22] Reddy TUK, Sindhu G, Rajesh S, Aruna B, Rani KSS. Preparation and evaluation of herbal hair oil. Indo Am. j. pharm. Sci 2017; 4(06): 1540-6.

[23] Sumangala BK, Kalpana P, Aishwarya T, Krithika G. Evaluation of herbal formulations on fungal pathogens of plants: A case study. Acta Scientific Agriculture 2019; 3: 122-4.

[http://dx.doi.org/10.31080/ASAG.2019.03.0734]

[24] Viswanad V, Aleykutty NA, Jayakar B, Zacharia SM, Thomas L. Development and evaluation of antimicrobial herbal formulations containing the methanolic extract of Samadera indica for skin diseases. J Adv Pharm Technol Res 2012; 3(2): 106-11. [http://dx.doi.org/10.4103/2231-4040.97285] [PMID: 22837958]

\section{(C) 2021 Tiwari et al.}

This is an open access article distributed under the terms of the Creative Commons Attribution 4.0 International Public License (CC-BY 4.0), a copy of which is available at: https://creativecommons.org/licenses/by/4.0/legalcode. This license permits unrestricted use, distribution, and reproduction in any medium, provided the original author and source are credited. 